\title{
QUEEN'S
UNIVERSITY
BELFAST
}

\section{The impact of psychoeducational interventions on the outcomes of caregivers of patients with heart failure: A systematic review \& Meta- analysis.}

Cassidy, L., Hill, L., Fitzsimons, D., \& McGaughey, J. (2020). The impact of psychoeducational interventions on the outcomes of caregivers of patients with heart failure: A systematic review \& Meta-analysis. International Journal of Nursing Studies, 114, [103806]. https://doi.org/10.1016/j.ijnurstu.2020.103806,

https://doi.org/10.1016/j.ijnurstu.2020.103806

\section{Published in:}

International Journal of Nursing Studies

\section{Document Version:}

Peer reviewed version

Queen's University Belfast - Research Portal:

Link to publication record in Queen's University Belfast Research Portal

\section{Publisher rights}

Copyright 2020 Elsevier.

This manuscript is distributed under a Creative Commons Attribution-NonCommercial-NoDerivs License

(https://creativecommons.org/licenses/by-nc-nd/4.0/), which permits distribution and reproduction for non-commercial purposes, provided the author and source are cited.

\section{General rights}

Copyright for the publications made accessible via the Queen's University Belfast Research Portal is retained by the author(s) and / or other copyright owners and it is a condition of accessing these publications that users recognise and abide by the legal requirements associated with these rights.

Take down policy

The Research Portal is Queen's institutional repository that provides access to Queen's research output. Every effort has been made to ensure that content in the Research Portal does not infringe any person's rights, or applicable UK laws. If you discover content in the

Research Portal that you believe breaches copyright or violates any law, please contact openaccess@qub.ac.uk. 


\section{Title}

The impact of psychoeducational interventions on the outcomes of caregivers of patients with heart failure: A systematic review and meta-analysis.

\section{Abstract}

Background: Heart failure is a global health care problem that causes a significant economic burden. Despite medical advancements, its prognosis remains poor as many patients with heart failure experience symptoms that negatively impact Quality of Life. Caregivers are often responsible for helping and supporting family members manage their heart failure symptoms at home. In addition to managing their own medical problems and maintaining social and personal lives, significant burden and stress can occur. At present, caregivers receive little guidance or information to support them in their caregiving role.

Objectives: This review aims to determine the impact of psychoeducational interventions on the outcomes of caregivers of heart failure patients.

\section{Design: Systematic review and meta-analysis.}

Data source: Five electronic databases: PsycINFO, Medline, CINAHL Plus, EMBASE and SCOPUS were searched from June 2007 to August 2019.

Review methods: The conduct and reporting of this review was based on the Cochrane Handbook for Systematic Reviews of Interventions and the Preferred Reporting Items for Systematic Reviews and Meta-Analyses. The Cochrane Risk of Bias Tool was used to assess the risk of bias among randomised controlled trials, and the Newcastle Ottawa Scale was used to assess risk of bias in one quasi-experimental study.

Results: Ten articles met the inclusion criteria, consisting of seven studies, with a total sample size of 953 participants. The pooled result from two studies, conducted in America and China, reported that psychoeducational interventions significantly reduced depression at six months' follow-up (SMD -0.82; $95 \% \mathrm{Cl}-1.17$ to $-0.47 ; \mathrm{p}=0.73, \mathrm{I}^{2}=0 \%$ ). The pooled result from two studies conducted in Sweden and Taiwan showed a significant improvement in heart failure knowledge at six months' follow-up (SMD $0.97 ; 95 \% \mathrm{Cl} 0.70$ to 1,$25 ; p<0.00001, \mathrm{I}^{2}=0 \%$ ). Finally, pooled results from three studies conducted in Sweden, China and Taiwan found a significant improvement in Quality of Life at 3 months' followup (SMD 0.25; 95\% Cl 0.25 to 0.48; $\mathrm{p}=0.03$ ), The three most common intervention components included: group based educational sessions, telemonitoring and telephone support, and written resources. 
Conclusions: There was no specific type of psychoeducational intervention found to have a significant impact on caregiver outcomes, as interventions were heterogeneous consisting of multiple components. Further research is needed to determine the effectiveness of individual and combined components to identify the ideal intervention format and design for caregivers of patients with heart failure.

Keywords: Heart failure; Caregivers; Psychoeducational interventions; Systematic review; Metaanalysis.

\section{Contribution of the paper}

What is already known about this topic?

- Heart failure is a global health care issue causing a severe symptom burden on patients and consequential increased responsibility on caregivers.

- Published data confirms that caregivers of patients with heart failure experience unmet needs. However, there is limited evidence on interventions designed specifically to meet these needs.

- Psychoeducational intervention studies that have been conducted involved patient and caregiver dyads, with outcomes demonstrating patient benefit.

\section{What this paper adds}

- This review found that psychoeducational interventions had a significant impact on caregiver outcomes, with results showing improved depression at 6 months, heart failure knowledge at 6 months, and quality of life at 3 months.

- The considerable heterogeneity across included study interventions highlights the need for a more standardised approach when designing future interventions for caregivers.

- Three main components were identified within the included interventions: group based educational sessions, telemonitoring and telephone support, and written resources.

\section{Introduction}

Heart failure (HF) has been described as a global health care problem, estimated to affect 26 million people worldwide, with over 5 million people in the United States of America (USA) and over half a million people in the United Kingdom (UK) $(1,2)$. Despite recent medical advancements, the prognostic outlook for many patients remains poor (3), with most experiencing frequent hospital admissions - causing a significant economic burden on global health care systems. It is estimated that $\mathrm{HF}$ is responsible for around $\$ 31$ billion of the health care expenditure in the USA, and $1-2 \%$ of 
the health care budget within Europe, with $60-70 \%$ of this cost incurred due to hospital admissions (4). HF has been referred to as the most malignant form of cardiovascular disease, as it causes a significant symptom burden for patients and survival rates are comparable with most cancers (5). Self-care behaviours and monitoring of symptoms are vital for improving patients' quality of life and clinical status, along with treatment plans involving complex medication regimens, frequent follow-up appointments and lifestyle changes $(6,7)$. Patients find it difficult to manage and monitor those symptoms of heart failure which often impact their physical and mental wellbeing (8).

Self-care is the performance of specific activities that enable patients' to manage their illness and maintain their health, and has been referred to as the cornerstone of heart failure management $(9,10)$. It is recommended as a key strategy within the European Society of Cardiology (ESC) 2016 guidelines for managing and treating heart failure (7). Caregivers frequently help patients with daily activities that support self-care, taking on various responsibilities alongside managing their own personal, medical and social lives $(11,12)$. Caregivers facilitate specific activities, such as: following diet restrictions, arranging hospital appointments, weighing the patient, navigating the healthcare system, providing information to clinicians at appointments and motivating the patient to improve selfcare (13). These activities help monitor patient symptoms, while reducing the risk of hospital readmissions and improving patients' quality of life $(6,14)$. However, evidence has found that caregivers of patients with HF can experience poor mental and physical health as a result of their caregiving responsibilities (15).

A recent study found that caregivers experienced high levels of burden, depression and anxiety that were linked to their caregiving role, as well as the health status of the patient. It was found that caregivers' level of burden was intensified when caring for a patient with depression and the level of burden was predicted by feelings of unpreparedness for their caregiving role (15). Caregivers often struggle with the uncertainty of HF, which can stem from the lack of information given from health care professionals concerning, for example, the patient's condition, signs and symptoms of HF, and what to expect now and in the future. Indeed, many caregivers have expressed the need for improved communication and information. The responsibility associated with the caregiving role can cause them to experience feelings of stress, anxiety, loneliness and depression (16). Qualitative research has found that caregivers often experience a lack of social support, and receive little guidance or support from health care professionals $(11,17)$. Many caregivers are in need of better social and emotional support as a way to help maintain a sense of normality (15). Better emotional and informational support, along with improved service provision, have been recommended to help alleviate the burden $(15,16)$.

Psychoeducational support has been recognised within ESC (2016) guidelines as a key component of heart failure management programmes to which caregivers should be involved (7). Psychoeducational interventions are gaining popularity and have shown positive effects on the wellbeing of caregivers of patients with psychosis, cancer and dementia (18,19,20). A psychoeducational intervention is defined as "a structured program that focuses on providing information about the care receiver's disease and about resources and services that can help 
caregivers manage specific disease-related problems" (21). There is limited evidence on the effectiveness of such interventions for caregivers of patients with heart failure. Previous systematic reviews have identified the needs of caregivers of patients with HF and the problems they experience due to their caregiving role $(22,23)$. An integrated review examined interventions focused on improving psychological outcomes in caregivers of patients with heart failure. The eight studies identified, published between 2005 and 2015, provided mixed results on a range of caregiver outcomes, with the most common interventions being: face-to-face, nurse led psychoeducational sessions; home visits and telephone calls (24). There was no attempt made to assess the effectiveness of these interventions, and it was concluded that due to the variation in outcome measures, format of interventions and length of follow-up, a meta-analysis was not possible. A systematic review that focused on intervention components, contexts and outcomes of dyadic HF selfcare interventions produced a similar conclusion (25). This review included twelve intervention studies, published between 2000 and 2016, and concluded that the dyadic interventions were heterogeneous, and it was therefore not possible to identify what type of intervention was most effective. The review focused on dyads, and self-care interventions, with the outcome of studies being patient related and the specific effects of the interventions on caregiver reported outcomes was not assessed.

To date no systematic review has assessed the impact of psychoeducational interventions on caregiver reported outcomes or identified what elements are effective. This review will determine what elements of a psychoeducational intervention are the most effective at improving the wellbeing of carers to help guide the development of a novel intervention. Furthermore, given the increasing clinical need for caregiver support and education, an up-to-date systematic review was warranted to inform the design of a future clinical trial. Therefore, the aim of this review is to determine the impact of psychoeducational interventions involving caregivers and patients with heart failure on the outcomes

of caregivers.

\section{Methods}

\subsection{Design}

We used the Cochrane Handbook for Systematic Reviews of Interventions to guide a systematic review examining the effects of psychoeducational interventions on caregivers of patients with heart failure (26). We also used the Preferred Reporting Items for Systematic Reviews and Meta-Analyses to guide the conduct and reporting of this review (27).

\subsection{Eligibility criteria}

Eligible studies followed a strict inclusion and exclusion criteria: 


\subsubsection{Participants}

Studies that included adult patients with heart failure and their caregivers, and studies that included caregivers solely were eligible. Caregivers were defined as someone who provided regular unpaid care for a family member, partner or friend who needs help because of their illness and cannot cope without their support (28). Studies that included paid or employed individuals (post-operative, hospice patients or caregivers) were excluded.

\subsubsection{Types of studies}

Randomised controlled trials (RCT) or quasi-experimental designs were included. Intervention studies were included as this meant that the effectiveness of the intervention was assessed. Noninterventional designs, including: qualitative, case-control as well as commentaries and conference abstracts were excluded.

\subsubsection{Types of interventions}

Studies that used psychoeducational interventions involving either dyads consisting of heart failure patients and their caregivers or caregivers were included. A psychoeducational intervention was defined as interventions that provided any form of information and support to caregivers about the patient's disease process, resources and services to help caregivers manage disease-related problems (21).

\subsubsection{Types of outcomes}

Studies were included that measured any of the following caregiver outcomes: caregiver burden, depression, heart failure knowledge or quality of life (QoL). Studies that reported patient outcomes only were excluded.

\subsection{Search methods}

A systematic search of articles published in English between June 2007 and August 2019 was applied across five electronic databases: PsycINFO, Medline, CINAHL Plus, EMBASE and SCOPUS. We used multi-purpose searches for text words and MESH terms including: caregiver, intervention study and heart failure. Key words under each concept were combined using 'OR' and then the three concepts were grouped with 'AND'. The search terms and key words used were: 1. Caregiver: ("carer" OR "informal caregiver" OR "informal carer" OR "family member" OR "dyad") AND 2. Intervention study: ("intervention program" OR "intervention" OR "randomised control trial") AND 3. Heart failure: ("advanced heart failure" OR "chronic heart failure" OR "end stage heart failure"). A MEDLINE search strategy was developed with assistance from a medical librarian and was translated into each 
database using appropriate controlled vocabulary. An overview of the search strategy used is shown in Appendix 1.

\subsection{Study selection}

Studies were screened by two reviewers (LC \& JM) for relevance based on title and abstract. Full texts of potentially relevant articles were examined for eligibility based on the inclusion and exclusion criteria. We used our definition of psychoeducational interventions as part of our criteria for study inclusion. Any disagreements or discrepancies about inclusion of studies were resolved via discussion with a third (LH) and fourth reviewer (DF).

\subsection{Data extraction}

Data was extracted using an adapted version of the Cochrane Effective Practice and Organisation of Care data extraction form (29). Key data was extracted in tabular form and reviewed by a second independent author (JM), with discrepancies resolved via discussion. Information was extracted from each study on: design, study location, intervention setting (in-hospital or remote), intervention delivery and components, comparison groups (control group or standard care), participant characteristics, outcome measures, time points and results. These details were reported narratively to allow for comparisons to be made across all interventions.

\subsection{Risk of bias}

The Cochrane Risk of Bias Tool was used to critically appraise the quality of included randomised controlled trials by two independent authors (LC \& JM) (29). This assessed the quality of studies across six domains: sequence generation, allocation concealment, blinding of participants/ personnel/ outcomes, incomplete outcome data, selective outcome reporting and other sources of bias. The authors graded each risk of bias item as high, unclear or low risk of bias (Fig. 2). The Newcastle Ottawa Scale (NOS) was used to assess the quality of included non-randomized studies based on a star system that evaluates three domains: selection of study groups, comparability of groups and ascertainment of exposure. High quality choices were identified with a star, and a maximum of one star was allocated for each item within the 'Selection' and 'Exposure' categories and a maximum of two stars for 'Comparability'. A maximum of 9 stars can be awarded in total and a score of 7-8 was considered high quality, and studies with less than 5 stars were identified as high risk of bias $(30,31)$.

\subsection{Data synthesis}

Review Manager Software Version 5.3 was used to carry out a meta-analysis; forest plots were generated and pooled estimates were reported on the impact of psychoeducational interventions on caregiver reported outcomes (32). This was performed when studies reported on the same outcome, 
with the same follow-up period and sufficient data, using the random effects model. When pooling of data was not possible the findings were reported narratively. The treatment effect was measured using standard mean difference (SMD) and 95\% confidence intervals (Cls) were calculated for all data that were analysed. The $\mathrm{I}^{2}$ statistic was used to assess the statistical heterogeneity between selected studies, to detect consistency or inconsistency of findings. $I^{2}$ values of $25 \%, 50 \%$ and $75 \%$ indicated low, moderate or high total variation across studies (33). The quality of the evidence of each outcome was assessed using the GRADE criteria by one independent author (LC). This rated risk of bias, consistency, imprecision, indirectness, and publication bias as high, moderate or low (34). Studies were downgraded based on the following: study design, inconsistency across studies, indirect evidence across studies, imprecise results and publication bias. The GRADEpro Guideline Development Tool was used to produce a 'Summary of Findings' table (See Appendix 2).

\section{Results}

\subsection{Search outcomes}

A total of 867 articles were identified and all were exported to a reference management programme which removed 326 duplicates, with 541 articles remaining that were screened for eligibility (Fig. 1). Abstracts and titles were screened in relation to inclusion/exclusion criteria, resulting in 504 exclusions and leaving 37 articles. The remaining 37 articles were reviewed in full text by two reviewers (LC \& JM) and any disagreement was resolved by a third (LH) and fourth reviewer (DF). A total of 27 articles were excluded, with main reasons being: unsuitable patient population $(n=6)$, unsuitable intervention ( $n=11)$, and caregiver outcomes not explored $(n=10)$. In total, 10 articles (consisting of 7 studies) were included in this review.

\section{<Fig. 1. Insert>}

\subsection{Quality of studies}

Fig. 2 provides a summary of the risk of bias across included randomised controlled trials. All studies reported an adequate method of "random sequence generation" and provided reasons for attrition rates. In individual trials there was unclear allocation concealment $(35,36)$, and blinding of participants and personnel $(35,37)$, with high risk of bias in one study (38). It was unclear in one study whether outcome assessment was blinded (35), and one study presented a high risk of bias as there was no outcome data reported for the control group (37). All articles provided reasons for exclusions and attrition rates, with 9 out of 10 providing a flowchart to illustrate this. One study assessed using the Newcastle Ottawa Scale (NOS) was rated as four stars, therefore representing high risk of bias (39). A high risk of bias was calculated due to the lack of randomisation used, and poor reporting methods. 
Seven studies reported in ten articles were included, six were randomized controlled trials and one was a quasi-experimental study (39). Three articles published a range of results from the same RCT $(38,40,41)$, and two articles produced results based on another RCT $(35,42)$. Studies were conducted across five countries, including: Sweden $(n=2)(35,38)$, USA $(n=2)(36,37)$, China $(n=1)(43)$, Taiwan $(n=1)(39)$, and Thailand $(n=1)(44)$. Sample sizes ranged from 20 (36) to 369 (37) participants, with a total sample size of 953 participants. Five trials (reported in seven articles) included dyads, consisting of patients with HF and their caregivers (36-41), one included caregivers who lived outside the patient's home (37). Two trials (reported in three articles) focused on family caregivers solely $(35,42,43)$. The mean age of caregivers ranged from 41 to 70 years and the majority were female spouses or adult children. The mean age of patients ranged from 62 to 73 and the majority were male (see Table.1).

\subsection{Sociocultural aspects of carers}

There were country specific differences found between studies that may have affected the status of caregivers and their ability to participate in interventions. For example one study only accepted family caregivers who were able to pay for the Telehealth care intervention and the majority of these participants had a bachelor's degree, meaning that their participation was influenced by their economic and educational situation (39). Two studies included only caregivers who had access to an internet connection which may have had economic implications for inclusion $(37,39)$. Three studies stated that the interventions tested were adapted to suit the cultural context of that specific country indicating that including participants from a low economic status could have been related to increased levels of burden $(36,43,44)$. A further study included African American caregivers only and adapted their intervention strategies to suit the customs and traditions of this specific population (36). One study included adult children caregivers who were significantly younger than those from Western countries, often shared caring responsibilities between family members and experienced less burden than full-time carers (44).

\subsection{Intervention details}

The duration of interventions from the included studies ranged from 1 month (39) to 12 months (37) and follow-up periods ranged from one month (39) to twenty- four months (41). Five studies were conducted in hospitals $(35,38,39,43,44)$ and two in outpatient clinics $(36,37)$ (See Table. 1). Interventions varied in terms of duration and intensity, as six studies used multi-component interventions (38, 39, 43, 36, 37, 44), including face-to-face educational sessions ( $38,43,44)$, written study used one component of face-to-face educational sessions (35). 


\subsubsection{Educational sessions}

Four studies used face-to-face educational sessions in their interventions (35, 38, 43, 44). Two studies used group-based sessions $(35,43)$ and two used single sessions with dyads $(38,44)$. One study included eight participants in a group (35) and the other included ten (43). One study used sessions that were focused solely on heart failure self-care (44), whereas the remaining studies provided education on a range of topics including: medical aspects of HF, self-care, practical skills to help with managing heart failure, lifestyle modifications, nutrition and psychological aspects of the caregiver role. The intensity of the sessions ranged from one (44) to six sessions (35), with duration of each session between one $(38,43,44)$ to two hours $(35)$. Three studies provided participants with written resources to supplement the sessions $(38,43,44)$, and one provided CD-ROMs (38). Two studies used nurses to deliver the educational sessions $(38,44)$ and two were delivered by multidisciplinary teams made up of a range of different healthcare professionals $(35,43)$.

\section{<Fig.2 Insert>}

\subsubsection{Telemonitoring and telephone support}

Two studies used telemonitoring in their interventions $(37,39)$, which is known as using electronic means consisting of web-based or telephone-based systems to record and monitor patient physiological data at home (45). One study used an electronic device called the Telehealth device that allowed caregivers to measure patients' physical symptoms at home, which were uploaded, monitored and analysed by a HF specialist (39). Patients received 24-hour health education counselling and the opportunity to attend a medical referral service that was available 24-7 via telephone. A study used Interactive Voice Response (IVR) to administer self-management support calls to patients every week for 12 months, with caregivers receiving an email after each call describing the patient's symptoms and what they could do to help support self-management (37). It was not reported who administered the emails to caregivers. One study provided four weeks of posthospital coaching delivered via telephone, focusing on skills to assist with managing HF at home (36). Each dyad took part in four weekly coaching sessions delivered by a nurse interventionist, helping caregivers set up routines to follow at home to help with the management of HF.

\subsubsection{Written resources}

Five studies used written resources within their interventions $(36,37,38,43,44)$. These were described as: a guide, manual, brochure and printed guidelines all tailored towards the needs of caregivers. One study provided a brochure for caregivers that was developed by a multidisciplinary team with information on: medical aspects of HF, self-care, emotional distress and available coping resources (43). Another study provided caregivers with two guides designed by the American Heart Association, one that contained information on symptoms and the other focusing on staying healthy. They also 
gave caregivers a list of local support organisations, a book on low-sodium recipes and a book called Comfort At Home for Chronic Heart Failure: A Guide for Caregivers (36). Patients and caregivers within one study were provided with a manual that explained key aspects of learning to live with HF, with skills training on: role-modelling, problem-solving and self-monitoring skills (44). They also received a DVD with matching content and were guided to watch this after reading each chapter. Caregivers were provided with printed guidelines in one study, containing information on how to communicate in a positive and motivating way to patients about self-care and HF related health (37). One study provided booklets to dyads as part of their intervention, however there were no details given about the content of these booklets (38).

\section{<Table. 1. Insert>}

\subsection{Impact of intervention}

\subsubsection{Caregiver burden}

Caregiver burden was measured in five studies $(36,38,39,41,43)$, two of which used the 22-item Caregiver Burden Scale (CBS) $(38,41,46)$. One study used the Chinese version of the 22-item ZBI scale $(43,47)$, one used the Chinese version of the 28 -item Caregiver Burden Inventory CBI scale $(39,48,49)$ and one used a modified 17 -item scale $(36,50)$. All studies provided baseline data, one study provided 1 month follow-up data (39), two studies provided 3 month follow-up data $(38,43)$, two studies provided 6 month follow-up data $(36,43)$, one study provided 12 month follow-up data (38) and one provided 24 month follow-up data (41). A higher score across all scales meant a greater level of burden.

A meta-analysis (Fig. 3) of two studies measuring the impact of psychoeducational interventions on caregiver burden at 3 months $(38,43)$ found no significant improvement (SMD $-0.22 ; 95 \% \mathrm{Cl}-1.04$ to $0.61 ; p=0.0007)$, and a high level of heterogeneity between studies $\left(I^{2}=91 \%\right)$. A non-significant improvement in caregiver burden was also found between two studies measuring the impact of psychoeducational interventions on caregiver burden at 6 months $(36,43)(\mathrm{SMD}-0.29 ; 95 \% \mathrm{Cl}-0.87$ to $0.29, p=0.32)$, with a moderate level of heterogeneity between studies $\left(I^{2}=43 \%\right)(F i g .3)$

\section{<Fig. 3. Insert>}

Using GRADE summary of evidence, the quality of evidence was very low, and outcomes were downgraded due to high risk of bias, inconsistency, indirectness and imprecision. Two studies were excluded from the meta-analysis as follow-up periods and outcome measures were different. One study tested the impact of a psychoeducational intervention on caregiver burden at 24 month followup (41), and found no significant difference between the intervention group and control group $(p=$ 
0.803). Another tested the impact of a telehealth intervention on caregiver burden at 1 month followup (39), and found a significant reduction in caregiver burden within the intervention group compared to the control group $(p<0.001)$.

\subsubsection{Depression}

Six studies measured depression $(36,37,38,40,42,43)$. Three studies used different versions of the Center for Epidemiologic Studies Depression Scale (CES-D) $(36,37,43)$. Two studies used the 10item version $(36,37,51,52,53)$, and one study used the 20 -item Chinese version $(43,54)$. Two studies $(38,40)$ used the 21-item Beck Depression Inventory (BDI-II) $(55)$, and one (42) used the Hospital Anxiety and Depression Scale (HADS) (56). All studies provided baseline data, two provided 3 month follow-up data $(38,43)$, three provided 6 month follow-up data $(36,37,43)$, two provided 12 month follow-up data $(37,38)$ and one provided 24 month follow-up data $(40)$. A higher score across all scales indicated a higher level of depression.

A meta-analysis was conducted on two studies that measured the impact of psychoeducational interventions on depression at 3 month follow-up $(38,43)$. Fig. 4. shows a non-significant difference in depression found between intervention group and control group (SMD $-0.40 ; 95 \% \mathrm{Cl}-0.92$ to $0.13 ; \mathrm{p}=$ $0.14)$ and a high level of heterogeneity between studies $\left(I^{2}=79 \%\right)$. Two studies measured the impact of psychoeducational interventions on depression at 6 months and a significant reduction was identified $(36,43)(\mathrm{SMD}-0.82 ; 95 \% \mathrm{Cl}-1.17$ to $-0.47 ; \mathrm{p}=<0.00001)$ with no heterogeneity between studies $\left(I^{2}=0 \%\right)$ (Fig. 4).

\section{<Fig.4. Insert>}

Using GRADE, the quality of evidence was very low, and outcomes were downgraded for high risk of bias, inconsistency, and indirectness. Three studies were excluded due to lack of sufficient data and different follow-up periods. One study found no significant difference in depression between the intervention and control group at 6 months' following a psychoeducational intervention (42). One study found a significant reduction in depression at 6 months' $(p=<0.001)$, but a non-significant reduction at 12 months' $(p=0.031)(37)$. Another study found no significant reduction in depression at 24 month follow-up (40).

\subsubsection{Heart failure knowledge}

Two studies measured heart failure knowledge $(35,44)$, with the 15-item Dutch Heart Failure Knowledge Scale DHFKS used in one study $(44,57)$. A modified questionnaire was used in one study containing 13 questions that were based on a questionnaire that was originally developed for patients with chronic heart failure $(35,58)$. Both studies included baseline and 6 month follow-up data. Higher scores indicated higher levels of heart failure knowledge across both scales. 
A statistically significant improvement in heart failure knowledge was found in the intervention group compared to the control group (SMD 0.97; 95\% Cl 0.70 to 1,$25 ; p<0.00001$ ), with no heterogeneity between studies ( $\left.I^{2}=0 \%\right)$ (Fig. 5). Using GRADE, the quality of evidence was very low, downgraded for unclear risk of bias, inconsistency and indirectness.

\subsubsection{Quality of life}

Five studies measured quality of life $(38,40,42,43,44)$. Three studies used the Short Form (SF) 36 item scale $(38,40,43)$ that provided a summary of physical (PCS) and mental health (MCS) (59-61). One study used the Short-Form (SF) 12-item scale that also provided a summary of physical and mental health $(44,62)$. One study used the Cantril Ladder of Life, which measured overall quality of life on a scale of 1-10 (42,63). Baseline and post-intervention data was provided for all studies, three provided 3 month follow-up data $(38,43,44)$, three studies provided 6 month follow-up data $(42,43,44)$, one provided 12 month follow-up data (38), and one provided 24 month follow-up data (40). All scales stated that a higher score indicated a better level of quality of life.

A meta-analysis was conducted on three studies that measured the impact of psychoeducational interventions on QoL (MCS) at 3 months $(38,43,44)$. An improvement was found in QoL (MCS) (SMD $0.25 ; 95 \% \mathrm{Cl} 0.25$ to $0.48 ; \mathrm{p}=0.03)$, with low level of heterogeneity between studies $\left(\mathrm{I}^{2}=20 \%\right)$. Two studies measured the impact of psychoeducational interventions on QoL (MCS) at 6 months follow-up $(43,44)$. Fig. 6 demonstrates a non-significant difference between the intervention and control groups (SMD $0.35 ; 95 \% \mathrm{Cl}-0.23$ to $0.94 ; \mathrm{p}=0.24)$ and a high level of heterogeneity $\left(\mathrm{I}^{2}=79 \%\right)$.

$<$ Fig.6.Insert>

438

Using GRADE, the quality of evidence for outcomes was very low, downgraded for high risk of bias, inconsistency, indirectness and imprecision. One study was excluded from meta-analysis as sufficient data was unavailable, with patient and caregiver data presented as one unit (40). However, the study showed no significant differences in QoL (MCS) at 24 month follow-up for dyads $(p=0.601)$ following a psychoeducational intervention.

\section{Discussion}

447 This paper used a rigorous systematic process and high-quality methodology to identify and examine the impact of psychoeducational interventions on a number of caregiver related outcomes. Our review 
found a significant reduction in depression at 6 months, improved heart failure knowledge at 6 months and improved QoL (MCS) at 3 months. It is uncertain whether psychoeducational interventions improve caregiver outcomes as a result of considerable heterogeneity and low quality of evidence found across studies. The lack of statistical significance presented across outcomes could be accounted for by the differences between interventions, including variations in intensity, duration, format, and outcome measures. These findings are consistent with other reviews that have attempted to assess the impact of psychoeducational interventions for caregivers and patients with HF but have failed due to the high level of heterogeneity found between interventions $(9,10)$. Despite this, some similarities were identified across the seven trials.

458 Caregivers often lack confidence in their caregiving role due to a lack of information and education on HF and self-care $(15,22)$. All the interventions included education and information on self-care, although different resources and delivery strategies were used to deliver this information. They all aimed to guide and teach activities caregivers could do at home to help the patient's self-care through the provision of information and training of key skills. These included: weighing the patient, managing medications, following dietary recommendations and limiting fluid intake. Written resources were a key component used to provide caregivers with tailored information, and were used within five studies $(36,37,38,43,44)$. The most common topics covered in these resources included: medical aspects of $\mathrm{HF}$, symptom management, skills to help with self-care and communication. Educating caregivers on self-care and other aspects of HF is vital, as this can help them to support and confidently care for the patient at home (64). Lack of preparedness for caregiving is an issue that has been commonly mentioned within literature, and can contribute to poor quality of life for caregivers $(15,22,65,66)$.

A number of studies incorporated nurses, illustrating their key position in organising the overall care for patients with HF, especially following hospitalisation and during follow-up appointments that are usually held in nurse-led HF clinics (67). Specialist heart failure nurses provide information and advice during consultations and can help identify those caregivers in need of advice and support (67). Hospital discharge has been suggested as the most appropriate time to engage with caregivers, provide information to promote self-care and to equip them with essential skills to care for the patient at home (68). As hospital resources become scarcer, there is an increasing shift towards community or informal care for patients at home across many Western societies; therefore more caregivers are looking after chronically ill patients (69). It is important that caregivers are included in the planning of care and actively involved at hospital appointments and post-discharge (70). Providing caregivers with support and guidance can also help benefit patients, as an American study found after four weeks of post-discharge telephone coaching provided to patients and caregivers, patients experienced significantly less HF-related rehospitalisation's compared to those that received standard care (36). This result highlights that caregivers should be considered an integral part of the health care team (68).

Multidisciplinary involvement has been recognised as a key part of non-pharmaceutical interventions to support patients with HF and their family caregivers (7). Two studies used multidisciplinary teams to deliver group-based educational sessions, made up of a range of different health care professionals, 
including: a dietician, cardiologist, social worker, and cardiac nurse $(35,43)$. Interestingly, the study

489

490

491

492

493

494

495

496

497

498

499

500

501

502

503

504

505

506

507

508

509

510

511

512

513

514

515

516

517

518

519

520

521

522

523

524

525

that produced the most significant results overall - with improvements in caregiver burden, depression and QoL - used a multidisciplinary approach (43). Another study also showed a significant improvement in HF related knowledge using the same approach (35). Three studies used nurse-led educational sessions, and showed no significant improvements in any caregiver outcomes $(38,44)$. In summary, a multidisciplinary approach ensures that a broad range of needs are met using expert advice, and is therefore recommended due to caregivers having multiple needs requiring expertise from a number of different disciplines $(43,69)$.

Research on family caregiving is advancing (68); however, as this review indicates there are very few intervention studies that have been designed to improve outcomes for caregivers only. Most interventions involving caregivers have focused on improving patient outcomes (68), most commonly in terms of health care utilisation. Many studies testing family partnership interventions in heart failure have shown improvements in patient outcomes, including improved medication adherence, lowsodium diet adherence, perceived confidence and hospital admissions (70-74).

\subsection{Limitations}

We recognise that there are important limitations in this systematic review. The search strategy was restricted to English language and did not include grey literature which may have introduced publication bias (75). In addition, there was considerable heterogeneity across included studies in terms of design, resources used, delivery of the interventions, populations and outcomes measured, therefore making the presentation of meaningful data challenging (76). There were large variations between the patient and caregiver characteristics within the majority of studies, as well as cultural differences that may have affected the approach, content and delivery of some interventions. These limitations and the low quality of evidence, limits the robustness of the findings. This highlights the importance of future research to identify and agree standardised patient outcome measures or core outcome sets to represent the minimum which should be reported in randomised trials (77).

It was not possible to identify a specific component of the intervention that was most successful at improving outcomes for caregivers of patients with $\mathrm{HF}$, as the interventions implemented consisted of multiple components. We were unable to explore individual components of multi-component interventions, as included studies did not test the specific impact of each intervention component. Further research to understand what combined intervention components work and why is required (78). The results presented within the meta-analyses should be interpreted with caution as there were a small number of heterogeneous studies included which provided hierarchical data. The small numbers of included studies meant it was not possible to undertake a hierarchical modelling approach as recommended by Cochrane (29). This limits the robustness of the findings and it is recommended that a hierarchical approach is used when more studies become available to allow for a more robust meta-analysis to be performed. 
527 There is a need for improved communication and support within clinical settings, which could be aided

528 through improved nurse engagement with family caregivers and patients during clinic visits. Nurses

529 have a high degree of patient contact and are therefore vital in achieving optimal patient care.

530 Incorporating more support for caregivers into their role could help involve caregivers in the

531 coordination of care and improve their outcomes. In addition to this direct contact, caregivers are in

532 need of readily available resources that could be distributed within the clinical setting.

533

\section{5. Conclusion}

535 A number of international studies have shown significant improvements in, caregiver burden, 536 depression, heart failure knowledge and quality of life. Unfortunately, it remains difficult to state which 537 psychoeducational intervention and or component was the most successful at improving caregiver 538 outcomes. Overall, the quality of evidence was very low and there was notable heterogeneity across 539 studies; however, interventions had similar components, such as: educational sessions delivered by 540 multidisciplinary teams, telemonitoring and telephone support, and written resources. As a result, 541 future trials should include an element of group-based education, with involvement from 542 multidisciplinary teams and provision of information tailored specifically to carers. In addition, further 543 research is warranted into psychoeducational interventions that are adequately powered and are 544 targeted towards improving caregiver outcomes, with detailed process evaluation.

\section{Conflict of interest}

547 The authors have no conflicts of interest to disclose.

\section{Acknowledgements}

550 The authors acknowledge Dr Martin Dempster at Queen's University Belfast for his expert statistical 551 help and advice. We would also like to acknowledge Mrs Brenda Allen from the Queen's University 552 Belfast Medical Library, who helped to develop a search strategy for locating manuscripts that were 553 analysed. LC wrote the manuscript and received regular feedback from JMG, LH and DF.

Funding

556 PhD funding was provided by the Northern Ireland Chest Heart and Stroke organisation and Queen's 557 University Belfast. 
559 Ethical review

560 This review was not subject to ethical review.

561

562

563

564

565

566

567

568

569

570

571

572

573

574

575

576

577

578

579

580

581

582

583

584

585

586

587

588

589

590

591

592

593

16 | P a g e 
596 (1) Savarese G, Lund LH (2017) Global Public Health Burden of Heart Failure. Card Fail Rev, Apr; 3(1):7-11.

597 (2) Cowie MR. (2017) The heart failure epidemic: A UK perspective. Echo Research and Practice; 4(1):R15-R20.

598 (3) Mcmurray JJV, Adamopoulos S, Anker SD, Auricchio A, BÃhm M, Dickstein K, et al. (2012) ESC Guidelines 599 for the diagnosis and treatment of acute and chronic heart failure 2012. European Journal of Heart Failure 2012; 600 14(8):803-869.

601 (4) Ambrosy AP, Fonarow GC, Butler J, Chioncel O, Greene SJ, Vaduganathan M, et al. (2014) The global 602 health and economic burden of hospitalizations for heart failure: Lessons learned from hospitalized heart failure 603 registries. J Am Coll Cardiol ;63(12):1123-1133.

604 (5) Forsyth P, Richardson J, Lowrie R. (2019) Patient-reported barriers to medication adherence in heart failure in 605 Scotland. International Journal of Pharmacy Practice 2019;27(5):443-450.

606 (6) Welstand J, Carson A, Rutherford P. (2009) Living with heart failure: An integrative review. Int J Nurs Stud 607 2009;46(10):1374-1385.

608 (7) Ponikowski P, Voors A. 2016 Esc guidelines for the diagnosis and treatment of acute and chronic heart 609 failure: The Task Force for the diagnosis and treatment of acute and chronic heart failure of the European society 610 of cardiology (ESC): Developed with the special contribution of the heart failure association (HFA) of the ESC. 611 Russian Journal of Cardiology 2017;141(1):7-81.

612 (8) Zambroski CH, Moser DK, Bhat G, Ziegler C. Impact of symptom prevalence and symptom burden on quality 613 of life in patients with heart failure. European Journal of Cardiovascular Nursing 2005;4(3):198-206.

614 (9) Evangelista LS, Moser DK, Westlake C, Pike N, Ter-Galstanyan A, Dracup K. Correlates of fatigue in patients 615 with heart failure. Prog Cardiovasc Nurs 2008;23(1):12-17.

616 (10) Jiang Y, Shorey S, Seah B, Chan WX, Tam WWS, Wang W. The effectiveness of psychological 617 interventions on self-care, psychological and health outcomes in patients with chronic heart failure: A systematic 618 review and meta-analysis. Int J Nurs Stud 2018;78:16-25.

619 (11) Wingham J., Frost J., Britten N., Jolly K., Greaves C., Abraham C., et al. Needs of caregivers in heart failure 620 management: A qualitative study. Chronic Illness 201501 Dec 2015;11(4):304-319.

621 (12) Kang X, Li Z, Nolan MT. Informal caregivers' experiences of caring for patients with chronic heart failure: 622 Systematic review and metasynthesis of qualitative studies. J Cardiovasc Nurs 2011;26(5):386-394.

623 (13) Buck HG, Harkness K, Wion R, Carroll SL, Cosman T, Kaasalainen S, et al. Caregivers' contributions to 624 heart failure self-care: a systematic review. European Journal of Cardiovascular Nursing 2015;14(1):79-89.

625 (14) Riegel B, Lee CS, Dickson VV. Self care in patients with chronic heart failure. Nature reviews cardiology 626 2011;8(11):644.

627 (15) Mcllfatrick S, Doherty LC, Murphy M, Dixon L, Donnelly P, McDonald K, et al. The importance of planning for 628 the future: Burden and unmet needs of caregivers in advanced heart failure: A mixed methods study. Palliat Med 629 2018;32(4):881-890. 

Exacerbates the Support Needs of Current and Bereaved Caregivers in Advanced Heart Failure and Impedes Shared Decision-making. J Cardiovasc Nurs 2019;34(1):11-19.

633 (17) Bangerter LR, Griffin JM, Dunlay SM. Qualitative study of challenges of caring for a person with heart failure. 634 Geriatr Nurs 2018;39(4):443-449.

635 (18) Sin, J., Gillard, S., Spain, D., Cornelius, V., Chen, T. and Henderson, C., 2017. Effectiveness of 636 psychoeducational interventions for family carers of people with psychosis: A systematic review and meta637 analysis. Clinical Psychology Review, 56, pp.13-24.

638 (19) Tang, W.P., Chan, C.W., Leung, D.Y. and Chan, D.N., 2019. The effects of psychoeducational interventions 639 on caregivers of children with cancer: A meta-analysis of randomized controlled trials. Journal of Child Health 640 Care, p.1367493518814917.

641 (20) Kishita, N., Hammond, L., Dietrich, C.M. and Mioshi, E., 2018. Which interventions work for dementia family carers?: an updated systematic review of randomized controlled trials of carer interventions. International psychogeriatrics, 30(11), pp.1679-1696.

644 (21) Sorensen, S, Pinquart, M, Duberstein, P. How effective are interventions with caregivers? An updated meta645 analysis. Gerontologist 2002; 42: 356-372.

646 (22) Doherty LC, Fitzsimons D, Mcllfatrick SJ. Carers' needs in advanced heart failure: A systematic narrative 647 review. European Journal of Cardiovascular Nursing 2014;15(4):203-212.

648 (23) Grant JS, Graven LJ. Problems experienced by informal caregivers of individuals with heart failure: An 649 integrative review. Int J Nurs Stud 2018;80:41-66.

650 (24) Evangelista L.S., Stromberg A., DionneOdom JN. An integrated review of interventions to improve 651 psychological outcomes in caregivers of patients with heart failure. Current opinion in supportive and palliative 652 care 201601 Mar 2016;10(1):24-31.

653 (25) Buck H.G., Stromberg A., Chung M.L., Donovan K.A., Harkness K., Howard A.M., et al. A systematic review 654 of heart failure dyadic self-care interventions focusing on intervention components, contexts, and outcomes. Int $\mathrm{J}$ 655 Nurs Stud 2018;77:232-242.

656 (26) Higgins JP, Green S. Cochrane handbook for systematic reviews of interventions. : John Wiley \& Sons; 6572011.

658 (27) Moher D, Liberati A, Tetzlaff J, Altman DG. Preferred reporting items for systematic reviews and meta659 analyses: the PRISMA statement. J Clin Epidemiol 2009;62(10):1006-1012.

660 (28) NHS Who is considered a carer? Available from:https://www.england.nhs.uk/commissioning/comm661 carers/carers/ [Accessed 13 February 2020]

662 (29) Cochrane Effective Practice and Organisation of Care. EPOC Resources for review authors. 2017.

663 (30) Veronese N, Bano G, Bertozzo G, Granziera S, Solmi M, Manzato E, et al. Vitamin K antagonists' use and 664 fracture risk: Results from a systematic review and meta-analysis. Journal of Thrombosis and Haemostasis 665 2015;13(9):1665-1675. 

assessing the quality of case-control studies in meta-analyses. Eur J Epidemiol 2011;25(9):603-605.

(33) Higgins JPT, Thompson SG, Deeks JJ, Altman DG. Measuring inconsistency in meta-analyses. Br Med J 2003;327(7414):557-560.

(35) Löfvenmark C, Karlsson MR, Edner M, Billing E, Mattiasson A-. A group-based multi-professional education programme for family members of patients with chronic heart failure: Effects on knowledge and patients' health care utilization. Patient Educ Couns 2011;85(2):e162-e168.

(36) Piamjariyakul U, Werkowitch M, Wick J, Russell C, Vacek JL, Smith CE. Caregiver coaching program effect: Reducing heart failure patient rehospitalizations and improving caregiver outcomes among African Americans. Heart and Lung: Journal of Acute and Critical Care 2015;44(6):466-473.

(37) Piette J.D., Striplin D., Marinec N., Chen J., Trivedi R.B., Aron D.C., et al. A Mobile Health Intervention 679 Supporting Heart Failure Patients and Their Informal Caregivers: A Randomized Comparative Effectiveness Trial. Journal of medical Internet research 201510 Jun 2015;17(6):e142. study evaluating effects of education and psychosocial support to patients with heart failure and their partners. $J$ Card Fail 2012 May 2012;18(5):359-366.

(39) Chiang L-, Chen W-, Dai Y-, Ho Y-. The effectiveness of telehealth care on caregiver burden, mastery of stress, and family function among family caregivers of heart failure patients: A quasi-experimental study. Int $\mathrm{J}$ Nurs Stud 2012;49(10):1230-1242.

\section{7} (40) Liljeroos M., Agren S., Jaarsma T., Arestedt K., Stromberg A. Long term follow-up after a Randomized integrated educational and psychosocial intervention in patient-partner dyads affected by heart failure. PLoS ONE 2015;10(9) (pagination):Arte Number: e0138058. ate of Pubaton: 25 Se 2015.

(41) Liljeroos M., Agren S., Jaarsma T., Arestedt K., Stromberg A. Long-term effects of a dyadic psychoeducational intervention on caregiver burden and morbidity in partners of patients with heart failure: a randomized controlled trial. Qual Life Res 2017;26(2):367-379.

(42) Lofvenmark C., Saboonchi F., Edner M., Billing E., Mattiasson AC. Evaluation of an educational programme 2013;22(1-2):115-126.

(43) Hu X, Dolansky MA, Su Y, Hu X, Qu M, Zhou L. Effect of a multidisciplinary supportive program for family caregivers of patients with heart failure on caregiver burden, quality of life, and depression: A randomized controlled study. Int J Nurs Stud 2016;62:11-21. 
(45) Mathew J, Lail J, Chang AC, Jefferies JL. Outpatient Monitoring and Self-Care. In Heart Failure in the Child and Young Adult 2018 Jan 1 (pp. 755-772). Academic Press.

(46) ElmstÃ $¥ \mathrm{hl}$ S, Malmberg B, Annerstedt L. Caregiver's burden of patients 3 years after stroke assessed by a novel caregiver burden scale. Arch Phys Med Rehabil 1996;77(2):177-182.

(47) Zarit S, Orr NK, Zarit JM. The hidden victims of Alzheimer's disease: Families under stress. : NYU Press; 1985.

707

(48) Chou K, Jiann-Chyun L, Chu $\mathrm{H}$. The reliability and validity of the Chinese version of the caregiver burden inventory. Nurs Res 2002;51(5):324-331.

(49) Novak M, Guest C. Application of a multidimensional caregiver burden inventory. Gerontologist 1989;29(6):798-803.

(50) Bakas T, Pressler SJ, Johnson EA, Nauser JA, Shaneyfelt T. Family caregiving in heart failure. Nurs Res 2006;55(3):180-188.

713

(51) Torres E. Psychometric properties of the center for epidemiologic studies depression scale in African American and black Caribbean US adults. Issues Ment Health Nurs 2012;33(10):687-696.

715

(52) Irwin M, Artin KH, Oxman MN. Screening for depression in the older adult: criterion validity of the 10-item Center for Epidemiological Studies Depression Scale (CES-D). Arch Intern Med 1999;159(15):1701-1704.

(53) Kohout FJ, Berkman LF, Evans DA, Cornoni-Huntley J. Two shorter forms of the CES-D depression symptoms index. J Aging Health 1993;5(2):179-193.

719 (54) Radloff LS. The CES-D scale: A self-report depression scale for research in the general population. Applied psychological measurement 1977;1(3):385-401.

(55) Beck AT, Ward CH, Mendelson M, Mock J, Erbaugh J. An inventory for measuring depression. Arch Gen Psychiatry 1961;4(6):561-571.

723

(56) Zigmond AS, Snaith RP. The hospital anxiety and depression scale. Acta Psychiatr Scand 1983;67(6):361370 .

(57) van der Wal, M.H., Jaarsma, T., Moser, D.K. and van Veldhuisen, D.J., 2005. Development and testing of the Dutch heart failure knowledge scale. European Journal of Cardiovascular Nursing, 4(4), pp.273-277.

727 (58) Karlsson MR, Edner M, Henriksson P, Mejhert M, Persson H, Grut M, et al. A nurse-based management program in heart failure patients affects females and persons with cognitive dysfunction most. Patient Educ Couns 2005;58(2):146-153.

730

(59) Li L, Wang HM, Shen Y. Chinese SF-36 Health Survey: translation, cultural adaptation, validation, and normalisation. J Epidemiol Community Health 2003 Apr;57(4):259-263. 36 Health Survey in 10 countries: Results from the IQOLA Project. J Clin Epidemiol 1998;51(11):1159-1165. 

assumptions, reliability and construct validity across general populations in Sweden. Soc Sci Med 1995;41(10):1349-1358.

737 (62) Ware Jr JE, Kosinski M, Keller SD. A 12-Item Short-Form Health Survey: construction of scales and preliminary tests of reliability and validity. Med Care 1996:220-233.

(63) Cantril H. Pattern of human concerns. 1965.

(64) Liljeroos M, Agren S, Jaarsma T, Stromberg A. Dialogues between nurses, patients with heart failure and their partners during a dyadic psychoeducational intervention: a qualitative study. BMJ Open 2017;7(12):e018236.

(65) Gusdal AK, Josefsson K, Adolfsson ET, Martin L. Registered Nurses' perceptions about the situation of family caregivers to patients with heart failure - A focus group interview study. PLoS ONE 2016;11(8).

(66) Hagedoorn El, Paans W, Jaarsma T, Keers JC, van dS, Luttik ML. Aspects of family caregiving as addressed in planned discussions between nurses, patients with chronic diseases and family caregivers: A qualitative content analysis. BMC Nursing 2017;16(1).

(67) Hagedoorn El, Keers JC, Jaarsma T, van dS, Luttik MLA, Paans W. The association of collaboration between family caregivers and nurses in the hospital and their preparedness for caregiving at home. Geriatr Nurs 2019.

751

(68) Jaarsma $T$, van dW, Hjelmfors L, Stromberg A. Talking about palliative care in heart failure. European Journal of Heart Failure 2018;20(9):1348-1349.

(69) Callahan CM, Boustani MA, Unverzagt FW, Austrom MG, Damush TM, Perkins AJ, et al. Effectiveness of collaborative care for older adults with Alzheimer disease in primary care: A randomized controlled trial. J Am Med Assoc 2006;295(18):2148-2157.

(70) Akhondzadeh K, Ghezeljeh TN, Haghani H. The effect of the education program on the adherence intention to the dietary sodium restriction and the amount of sodium intake in patients with chronic heart failure. Iranian Red Crescent Medical Journal 2018;20.

(71) Deek H, Chang S, Newton PJ, Noureddine S, Inglis SC, Arab GA, et al. An evaluation of involving family caregivers in the self-care of heart failure patients on hospital readmission: Randomised controlled trial (the FAMILY study). Int J Nurs Stud 2017;75:101-111.

(72) Dunbar SB, Clark PC, Stamp KD, Reilly CM, Gary RA, Higgins M, et al. Family partnership and education interventions to reduce dietary sodium by patients with heart failure differ by family functioning. Heart Lung $\mathrm{J}$ Acute Crit Care 2016;45(4):311-318.

(73) Shao J-, Chen S-. Randomized control trial of a self-management intervention for heart failure older adults in Northern Taiwan. Collegian 2019;26(2):288-294.

767 (74) Stamp KD, Dunbar SB, Clark PC, Reilly CM, Gary RA, Higgins M, et al. Family partner intervention influences self-care confidence and treatment self-regulation in patients with heart failure. European Journal of 

Clinic journal of medicine, 75(6), p.431.

(76) Turner, R.M., Bird, S.M. and Higgins, J.P., 2013. The impact of study size on meta-analyses: examination of underpowered studies in Cochrane reviews. PloS one, 8(3), p.e59202.

774

(77) Comet Initiative, 2015. Core outcome measures in effectiveness trials. Online information available at http://www. comet-initiative. org.

776

(78) Craig P, Dieppe P, Macintyre S, Michie S, Nazareth I, Petticrew M. Developing and evaluating complex interventions: The new Medical Research Council guidance. Int J Nurs Stud 2013;50(5):587-592.

778

779

780

781

782

783

784

785

786

787

788

789

790

791 

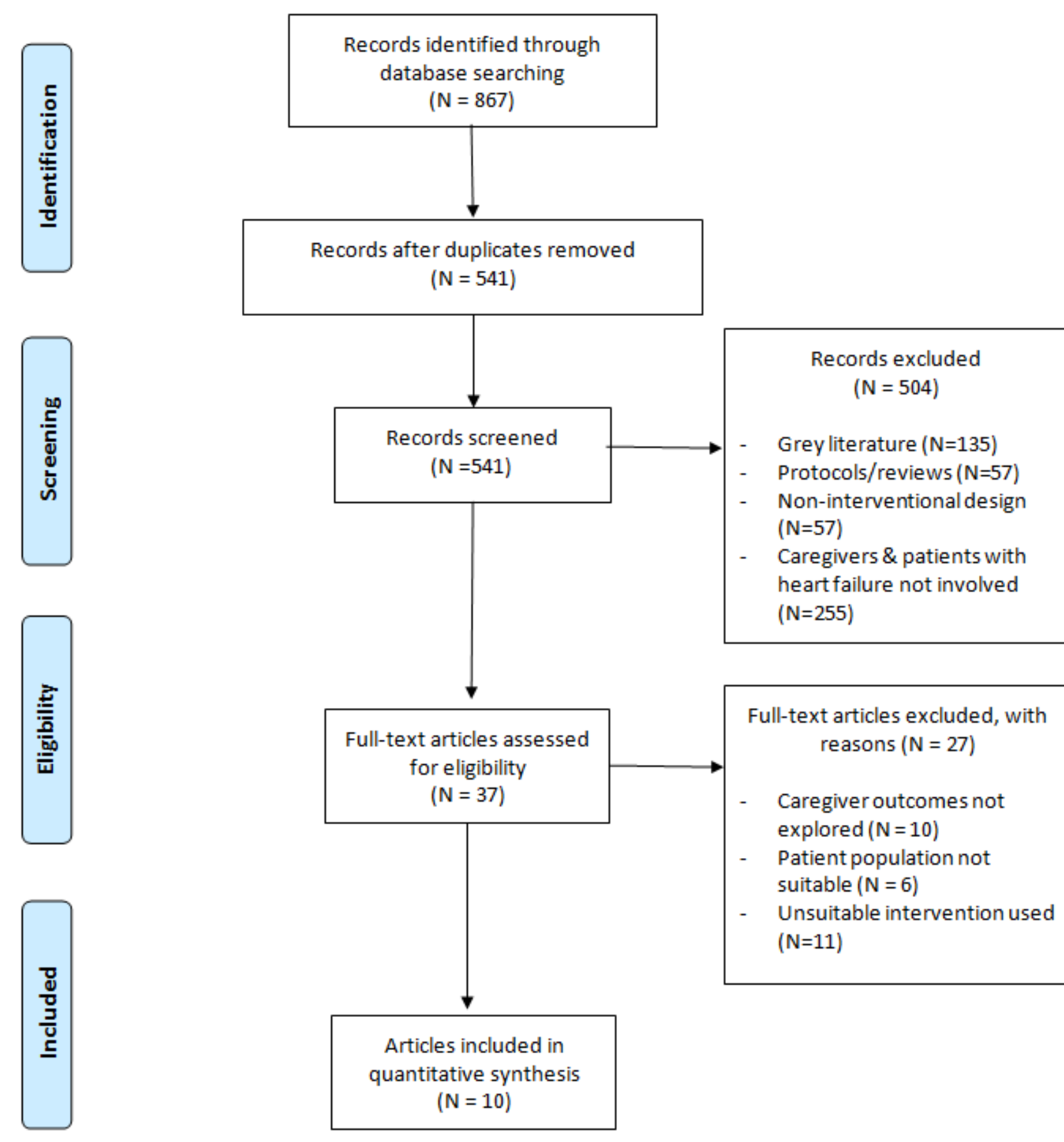

792

Fig. 1: PRISMA Flow diagram of study screening and selection (27) 


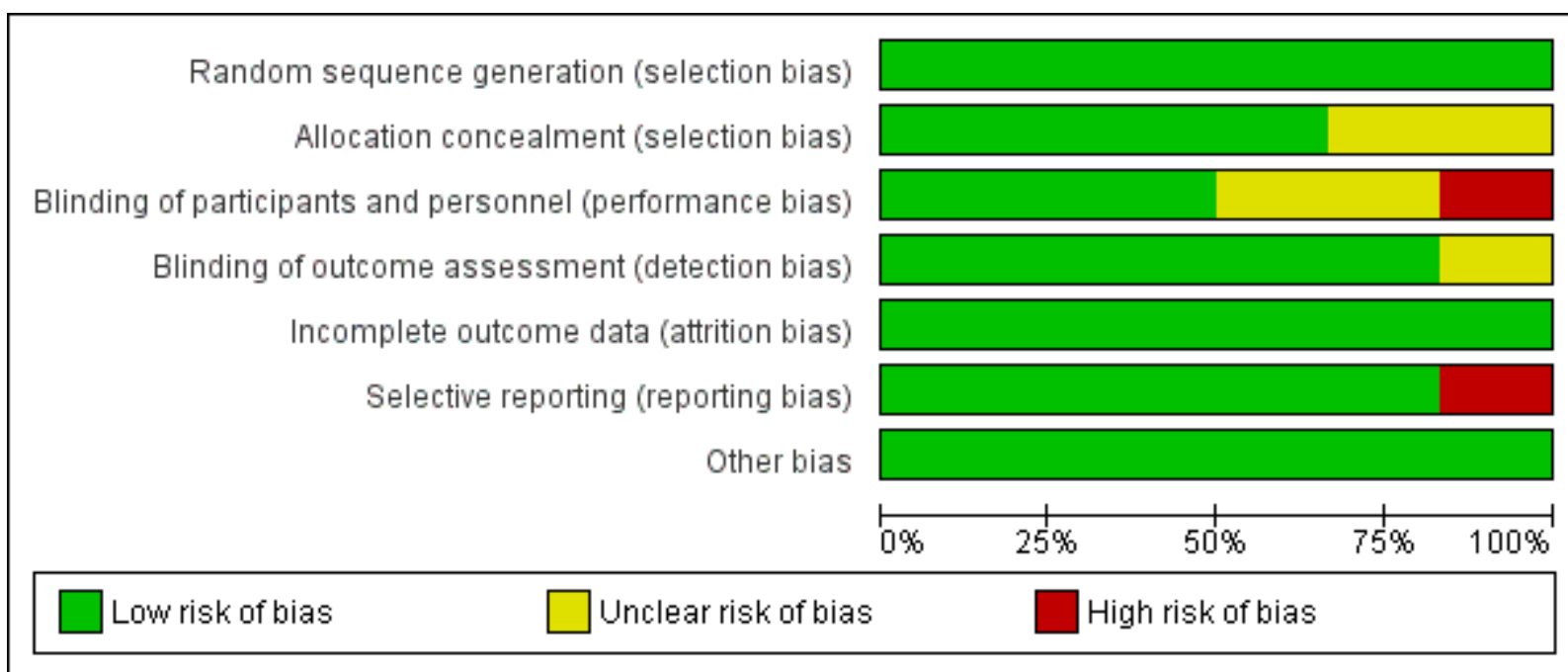

Fig 2: Risk of bias summary

800

801

802

803

804

805

806

807

808

809

810

811

812

813

814

815 
818 Table 1: Summary of included studies

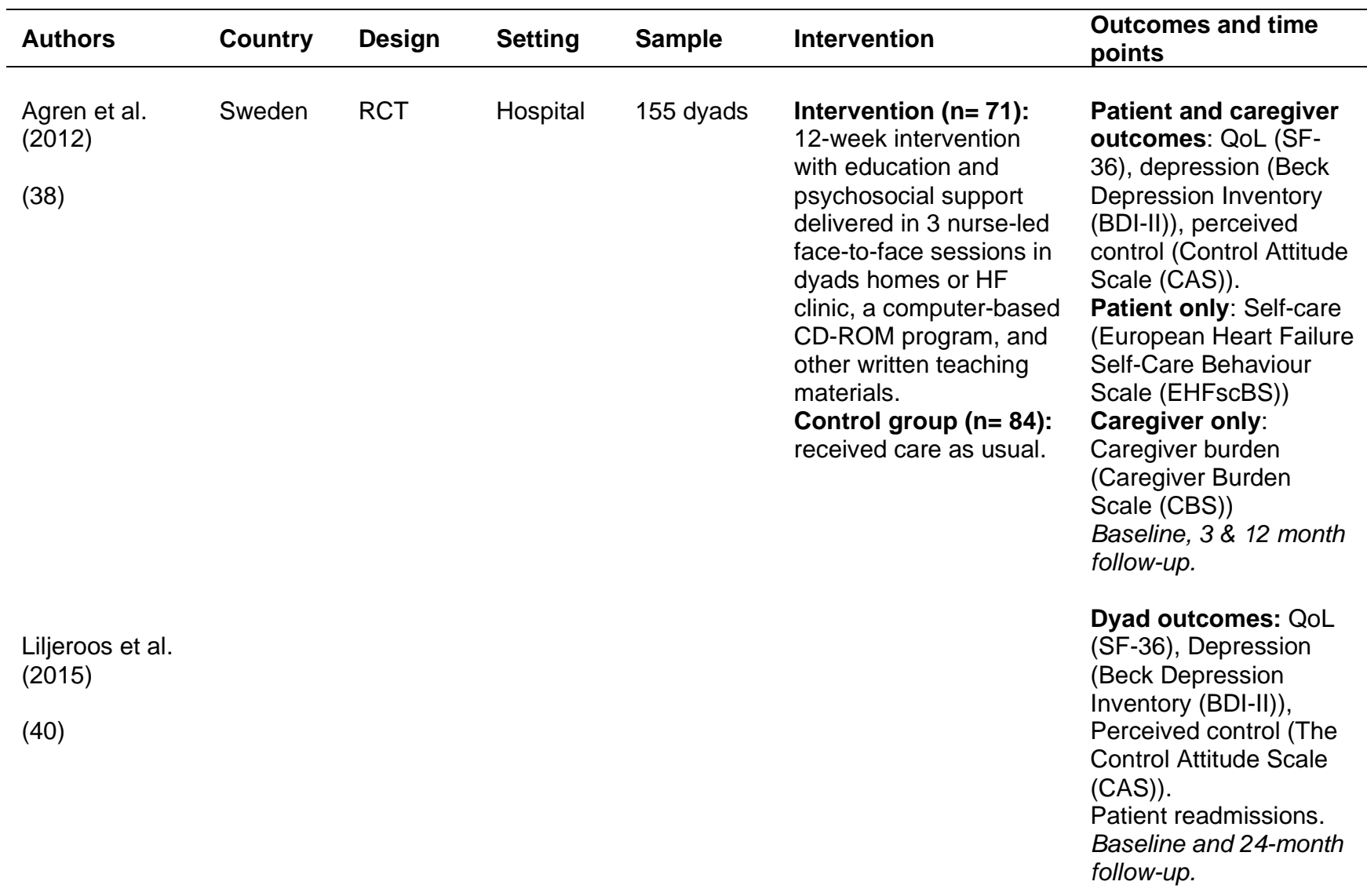

Liljeroos et al. (2017)

Chiang et al. (2012)
Taiwan Quasi-
experime
ntal

Hospital

63 dyads
Intervention $(\mathrm{n}=\mathbf{3 0}$

families): discharge

plan and telehealth device that caregivers were trained how to use along with 24-hour specialist education telephone counselling. Comparison group ( $n=30$ families): discharge planning from hospital.

\section{Caregiver outcomes:}

Caregiver burden

(Caregiver Burden

Scale (CBS)).

Baseline and 24-month follow-up.

Caregiver burden (Chinese version of the Caregiver Burden Inventory)

Before patient discharge \& 1-month follow-up
Results

No significant improvements in any carer outcomes.

Significant improvement in patient perceived control at 3-month follow-up, but not at 12-month follow-up.

No significant effects on any patientpartner dyads after 24 months.

No significant improvements in caregiver burden at 24-month follow-up.

Caregiver burden significantly improved in intervention group.
Hu et al.

(2016)

(43)
China

RCT

Hospita

118

caregivers
Intervention $(n=59)$ : 3month multidisciplinary program: brochure, $3 \mathrm{x}$ 60-minute support group sessions, 3x 30-
Caregiver burden (ZBI 22-item scale), Health related QoL (SF36), Depression (CES-D 20 -item scale)
Significant improvements in depression, caregiver burden \& mental QoL in 
minute peer group

support sessions \&

regular telephone

follow-up.

Control group $(n=59)$ :

usual care only.

Löfvenmark et Sweden RCT al. (2011)

(35)

Löfvenmark et

al. (2013)

(42)

\section{Piamjariyakul}

et al. (2015)

(36)

America $\mathrm{RCT}$
Hospital 128 family members

Intervention $(\mathrm{n}=65): 6$ support group sessions with a multidisciplinary period.

Control $(n=63)$ :

received information during hospitalisation according to routine hospital practice. team over 6 -month

Heart failure knowledge (modified questionnaire) Baseline, 2 weeks after intervention \& 6 months after.

QoL (Cantril Ladder of Life), Depression and anxiety (Hospital Anxiety and Depression Scale (HADS)), Health related QoL (SWEDQUAL).

Baseline, 2 weeks after intervention \& 6 months after. t clinic

ntervention ( $n=10): 4$ weeks of telephone coaching with nurse and two caregiving guides, list of support organisations, lowsodium booklet, pill organiser \& book for caregivers.

Standard care group $(n=10)$ : education and materials routinely given in hospital to patients

Piette et al. (2015)
America RCT

Outpatien 369 dyads t clinic
Caregiver burden (Caregiving burden of HF home care management 17-item) Depression (CES-D 10item scale)

Baseline \& 6 months

\section{Intervention group (mHealth+ CP) ( $n=189)$ : patients received weekly self- support calls for 12 months with notifications sent to clinicians and booklet for caregivers, plus emails sent to care- partners.}

\section{Comparison group} (Standard mHealth) $(n=180)$ : patients received weekly selfsupport calls for 12 months \& caregivers received a booklet.
Significant improvement in heart failure knowledge score in intervention group.
No significant improvements in anxiety and depression or QoL among the intervention group.
Significantly lower depression \& improvements in caregiver burdenmedium effect size in total caregiving burden score.

\section{Caregiver strain} (Caregiver strain index) Depressive symptoms (CES-D 10-item version)

Baseline, 6 \& 12 months
Significantly less caregiver strain \& depressive symptoms in intervention group. 


\begin{tabular}{|c|c|c|c|c|c|c|c|}
\hline $\begin{array}{l}\text { Srisuk et al. } \\
\text { (2016) } \\
(44)\end{array}$ & Thailand & $\mathrm{RCT}$ & Hospital & 100 dyads & $\begin{array}{l}\text { Intervention group } \\
(\mathrm{n}=50): 6 \text { month } \\
\text { programme: } 1 \text { face-to- } \\
\text { face education session, } \\
\text { manual, DVD \& } \\
\text { telephone follow-up. } \\
\text { Usual care group } \\
(\mathrm{n}=50) \text { : standard } \\
\text { medical \& nursing care } \\
\text { from hospital. }\end{array}$ & $\begin{array}{l}\text { Heart failure knowledge } \\
\text { (Dutch Knowledge } \\
\text { Scale: DHFKS) } \\
\text { General QoL } \\
\text { (caregivers) (SF-12 } \\
\text { Short-Form } 12 \text { health } \\
\text { survey) } \\
\text { Baseline, } 3 \text { \& } 6 \text { months }\end{array}$ & $\begin{array}{l}\text { Significantly higher } \\
\text { heart failure } \\
\text { knowledge scores in } \\
\text { intervention group. }\end{array}$ \\
\hline
\end{tabular}




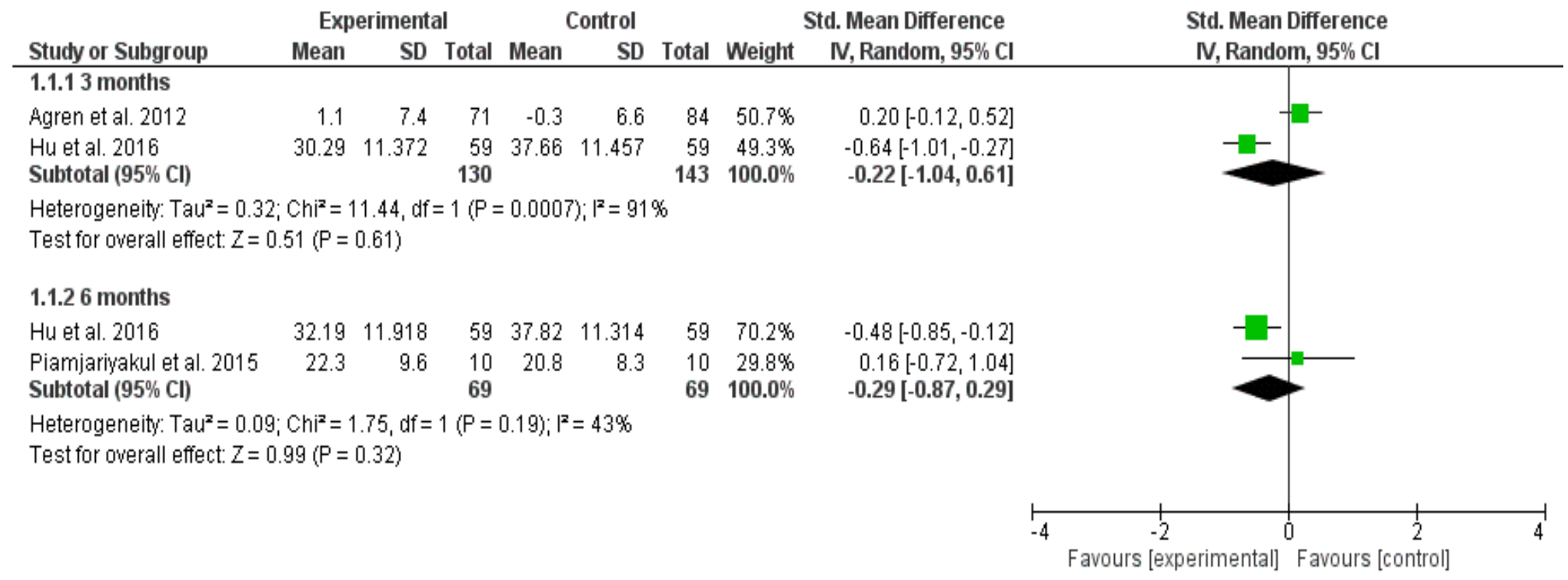

Fig. 3: Forest plot of the effect of psychoeducational interventions on caregiver burden at $3 \& 6$ months follow-up

839

840

841

842

843

844

845

846

847

848

849

850

851

852

853

854

855

856

857

858

859

860

861 


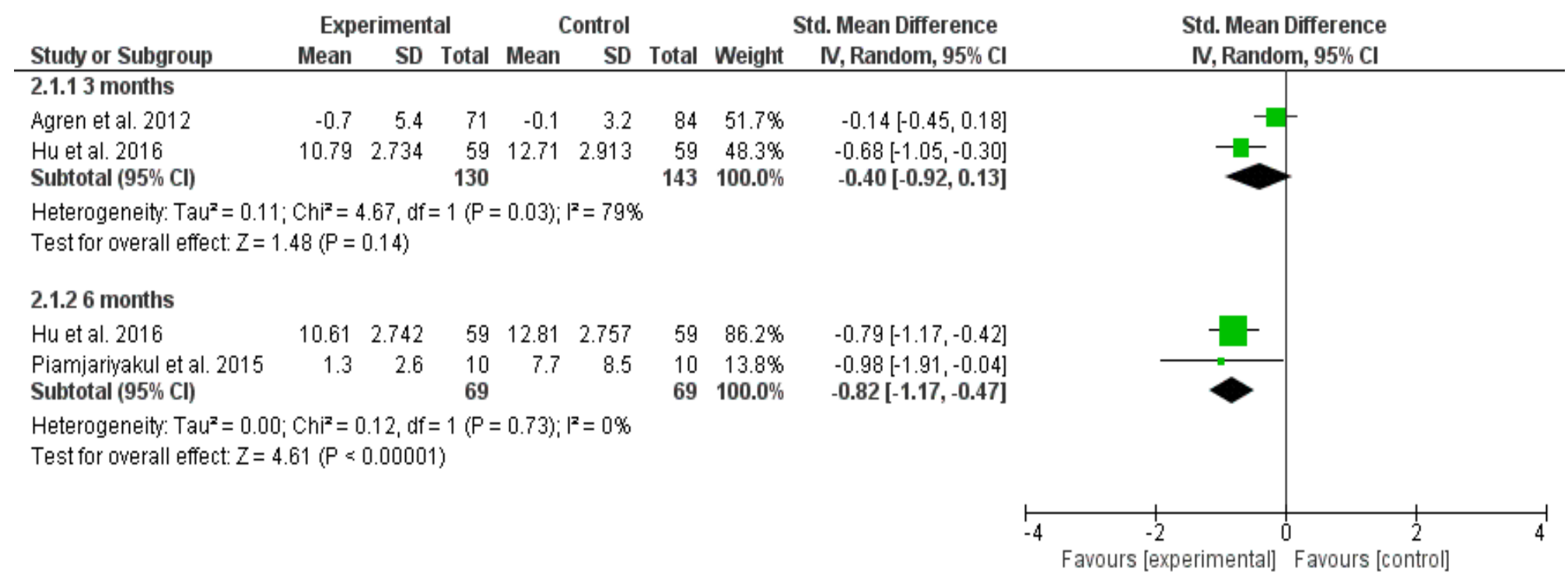

Fig. 4: Forest plot of the effect of psychoeducational interventions on depression at $3 \& 6$ months follow-up

862

863

864

865

866

867

868

869

870

871

872

873

874

875

876

877

878

879

880

881

882

883

884 
Experimental Control Std. Mean Difference Std. Mean Difference

Study or Subgroup Mean SD Total Mean SD Total Weight IV, Random, $95 \% \mathrm{Cl}$

IV, Random, $95 \% \mathrm{Cl}$

$\begin{array}{lllllllll}\text { Löfvenmark et al. } 2011 & 16.5 & 1.3 & 65 & 14.8 & 1.8 & 63 & 54.8 \% & 1.08[0.71,1.45\end{array}$

Srisuk et al. 2016

$\begin{array}{lllllll}12 & 2.84 & 50 & 9.6 & 2.84 & 50 & 45.2 \%\end{array}$

$0.84[0.43,1.25]$

Total $(95 \% \mathrm{Cl})$

115

$113100.0 \%$

Heterogeneity: Tau $^{2}=0.00 ; \mathrm{Chi}^{2}=0.73, \mathrm{df}=1(\mathrm{P}=0.39) ; \mathrm{I}^{2}=0 \%$

Test for overall effect: $Z=6.91(P<0.00001)$

$0.97[0.70,1.25]$

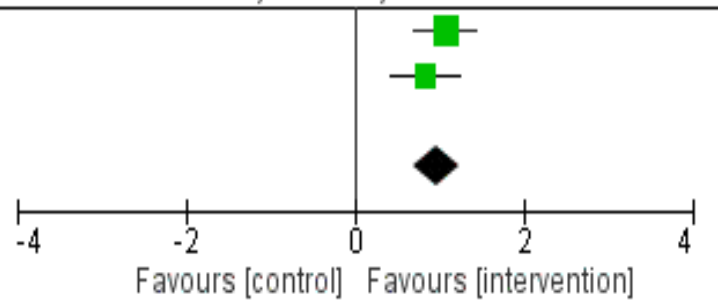

Fig. 5: Forest plot of the effect of psychoeducational interventions on heart failure knowledge at 6 month follow-up 885

886

887

888

889

890

891

892

893

894

895

896

897

898

899

900

901

902

903

904

905

906

907

908

909

910

911

912

913 
